\title{
Encapsulation narrows excitonic homogeneous linewidth of exfoliated MoSe 2 monolayer
}

\author{
Eric W. Martin ${ }^{1, *}$, Jason Horng ${ }^{1}$, Hanna G. Ruth ${ }^{1}$, Eunice Paik ${ }^{1}$, Michael-Henr Wentzel ${ }^{1}$, \\ Hui Deng ${ }^{1}$, and Steven T. Cundiff ${ }^{1}$ \\ ${ }^{1}$ Department of Physics, University of Michigan, Ann Arbor, MI 48109-1040, USA
}

\begin{abstract}
We use collinear multidimensional coherent spectroscopy to measure van der Waals structures with a nearly diffraction-limited spot size. Encapsulation by boron nitride narrows the homogeneous and inhomogeneous linewidths of excitonic resonances in $\mathrm{MoSe}_{2}$.
\end{abstract}

Monolayer van der Waals crystals are a class of materials with a growing interest among researchers and the potential to transform future electronics. These atomically thin layered materials with varying electronic properties can be stacked into heterostructures with synergistic benefits [1]. A subset of these materials are the semiconducting monolayer transition metal dichalcogenides (TMDCs), which have a direct band gap that makes their electronic transitions optically accessible. These materials are therefore useful for optoelectronic applications.

TMDCs are known to have widely varying excitonic linewidths that depend on the local environment, excitation source, and sample history (i.e. temperature cycles and exposure to laser radiation). Many of these inconsistencies have been remedied by encapsulation of the monolayer in hexagonal boron nitride $(\mathrm{hBN})$, which both controls the dielectric environment and protects the TMDC. Encapsulation has also led to photoluminescence linewidths that approach previous homogeneous linewidth measurements of nonencapsulated samples [2].

We use multidimensional coherent spectroscopy (MDCS) to compare the neutral exciton lineshapes of fully hBN-encapsulated molybdenum diselenide $\left(\mathrm{MoSe}_{2}\right)$ monolayer samples and non-encapsulated samples. We observe that both the inhomogeneous and homogeneous linewidths of the encapsulated samples are significantly narrowed in comparison to the non-encapsulated flakes. This narrowing is determined by a thorough power and temperature dependence of these various materials. We also report some negative attributes of the non-encapsulated samples that the protective hBN encapsulation prevents: we measure lasting broadening of the homogenenous linewidth of nonencapsulated samples by relatively weak $<40 \mu \mathrm{W}$ laser radiation, and we confirm the large sample dependence of their linewidths [3].

MDCS is a powerful technique that has been used to measure homogeneous lineshapes in the presence of inhomogeneity in a wide variety of physical systems [4] including TMDCs [5]. Standard MDCS relies on k-vector selection, which requires the creation of a

\footnotetext{
* Corresponding author: ewmartin@umich.edu
} 
density grating with a, typically, 15-50 $\mu \mathrm{m}$ spot size. We have developed a technique presented in [6] that uses heterodyne detection capable of measuring a radiated MDCS signal from a diffraction limited $<1 \mu \mathrm{m}$ spot. With the capability to acquire MDCS spectra from a diffraction limited spot, we are able to measure lineshapes of high quality prototypical TMDC heterostructures that are not currently produced on large spatial scales.

In Figures $1 \mathbf{a}$ and $\mathbf{b}$ we present images of two of the samples that we have measured using our collinear MDCS technique. These samples are (a) exfoliated $\mathrm{MoSe}_{2}$ on sapphire and (b) exfoliated $\mathrm{MoSe}_{2}$ encapsulated with a $123 \mathrm{~nm}$ layer of $\mathrm{hBN}$ on the bottom and a 24 $\mathrm{nm}$ layer on top (measured with atomic force microscopy), also on a sapphire substrate. In Fig. 1c, we plot linear reflectivity of the neutral exciton in these samples. Reflectivity measurements show linewidths that are broadened by inhomogeneity, and therefore cannot resolve homogeneous properties. In Fig. 1d and e we plot MDCS spectra of these nonencapsulated and encapsulated samples, respectively. These plots show the amplitude of the correlation between absorption (the $\omega_{\tau}$ axis) and emission (the $\omega_{t}$ axis). Individual oscillators absorb and emit along the plotted diagonal line, while coupling between oscillators appears off the diagonal. Thus, the lineshape of a slice along the cross-diagonal roughly corresponds to the homogeneous lineshape. In the limit of strong inhomogeneity, this lineshape is the square root of a Lorentzian. For similar inhomogeneous and homogeneous linewidths, the two are coupled, and we simultaneously fit the diagonal and cross-diagonal slices [7]. From these two spectra measured at $6 \mathrm{~K}$ with low power $(0.4$ $\mu \mathrm{W} /$ beam), it is evident that encapsulation has narrowed both the cross-diagonal (roughly homogeneous) and diagonal (roughly inhomogeneous) linewidths.

We acquire multidimensional spectra of several samples at several temperatures, and at each temperature we acquire multidimensional spectra for a range of excitation powers. As the excitation density (beam power) is increased, exciton-exciton scattering induces a broadening of the homogeneous linewidth that scales linearly with power. We plot the results of these measurements in Fig. 1f for a selection of the samples we have measured. Each data point represents the extrapolated zero-power homogeneous linewidth of the each sample at the plotted temperature. Since the acoustic phonon density increases linearly with temperature, we fit the temperature dependence to determine the zero-temperature (and zero-power) homogeneous linewidths of these samples, which defines $\gamma_{0}$. For the narrowest non-encapsulated sample $\gamma_{0}=0.42 \pm 0.05 \mathrm{meV}$, where the coherence time is defined $\mathrm{T}_{2}=\hbar /$ $\gamma_{0}=1.57 \mathrm{ps}$. However we find tremendous variability between samples with some samples exhibiting linewidths more than twice as large. Furthermore we measure significant broadening of the homogeneous linewidth after just a single temperature cycle. For the plotted encapsulated samples we measure an average $\gamma_{0}=0.26 \pm 0.02 \mathrm{meV}$ with corresponding $\mathrm{T}_{2}=2.53 \mathrm{ps}$. These samples have been measured after multiple temperature cycles with no change to their linewidths.

We thus demonstrate that encapsulation narrows the inhomogeneous and homogeneous linewidths of exfoliated $\mathrm{MoSe}_{2}$. We also demonstrate that applications requiring sample consistency necessitate the encapsulation of those samples. For a radiatively limited homogeneous linewidth, we discuss the scaling of each sample's linewidth resulting from radiative coupling and dielectric screening. Since we are still not sure which effect dominates, this experimental study will help to understand the microscopic behavior of TMDCs. 
a
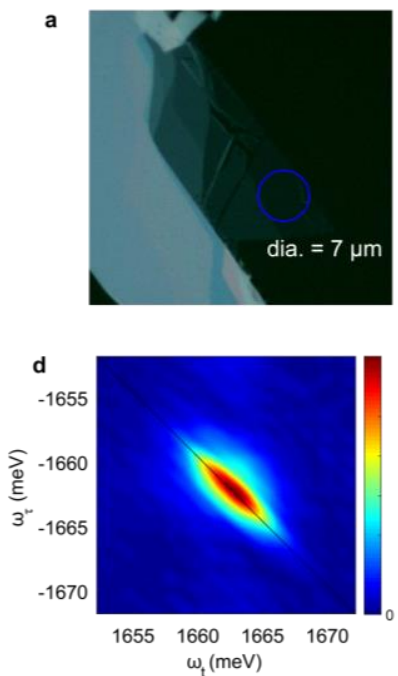

b
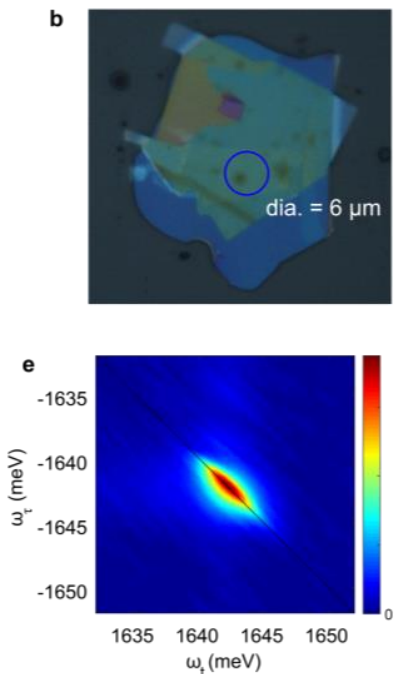
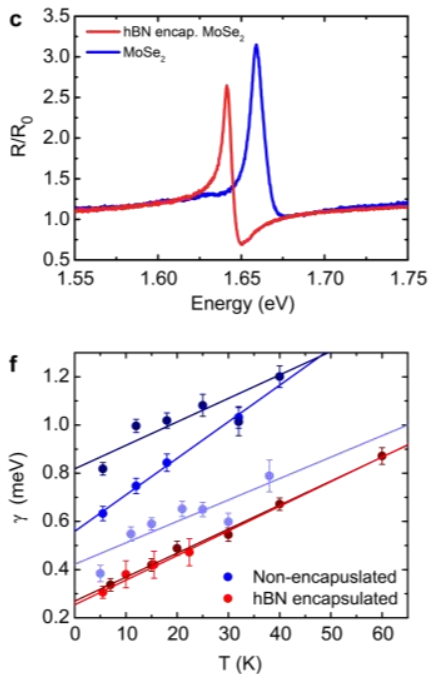

Figure 1: a Optical image of mechanically exfoliated $\mathrm{MoSe}_{2}$ flake on sapphire. b Image of boron nitride encapsulated $\mathrm{MoSe}_{2}$ on sapphire having a $<6 \mu \mathrm{m}$ area for measurement. $\mathbf{c}$ Linear reflectivity measurements reveal narrowing of the linewidth with encapsulation and can be used to estimate the oscillator strength of the resonance. Linear measurements cannot be used to determine the homogeneous linewidth. d MDCS is used to extract the homogeneous linewidth (roughly the crossdiagonal linewidth) of $\mathrm{MoSe}_{2}$ on sapphire in the presence of inhomogeneity (evidenced by the resonance broadening along the diagonal). e MDCS measurement of encapsulated $\mathrm{MoSe}_{2}$ reveals a narrowing of both the homogeneous and inhomogeneous linewidths. f MDCS measurements are made for a range of excitation densities at each plotted temperature. Therefore each point indicates the extrapolated zero-power linewidth for removing exciton-exciton scattering. We fit these points to extrapolate to the zero-temperature linewidth for removing exciton-phonon scattering. Blue points indicate non-encapsulated samples, where each shade of blue corresponds to a different sample. Red points indicate encapsulated samples.

\section{References}

1. K. S. Novoselov, A. Mishchenko, A. Carvalho, and A. H. Castro Neto, Science 353 (2016)

2. F. Cadiz, E. Courtade, C. Robert, G.Wang, Y. Shen, H. Cai, T. Taniguchi, K.Watanabe, H. Carrere, D. Lagarde, M. Manca, T. Amand, P. Renucci, S. Tongay, X. Marie, and B. Urbaszek, Phys. Rev. X 7, 021026 (2017)

3. T. Jakubczyk, V. Delmonte, M. Koperski, K. Nogajewski, C. Faugeras, W. Langbein, M. Potemski, and J. Kasprzak, Nano Letters 16, 5333-5339 (2016)

4. S. T. Cundiff and S. Mukamel, Phys. Today 66, 44 (2013)

5. G. Moody, C. Kavir Dass, K. Hao, C. H. Chen, L. J. Li, A. Singh, K. Tran, G. Clark, X. Xu, G. Berghauser, E. Malic, A. Knorr, and X. Li, Nat. Commun. 6, 8315 EP (2015)

6. E. W. Martin and S. T. Cundiff, Phys. Rev. B 97, 081301 (2018)

7. M. E. Siemens, G. Moody, H. Li, A. D. Bristow, and S. T. Cundiff, Opt. Express 18, 17699-17708 (2010) 